\title{
Universal Surface Engineering of Transition Metals for Superior Elec- trocatalytic Hydrogen Evolution in Neutral Water
}

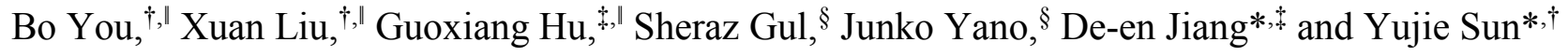 \\ ${ }^{\dagger}$ Department of Chemistry and Biochemistry, Utah State University, Logan, Utah 84322, United States \\ *Department of Chemistry, University of California, Riverside, California 92521, United States. \\ $\S$ Molecular Biophysics and Integrated Bioimaging Division, Lawrence Berkeley National Laboratory, Berkeley, California \\ 94720, United States.
}

Supporting Information Placeholder

\begin{abstract}
The development of low-cost hybrid water splitting-biosynthetic systems that mimic natural photosynthesis to achieve solar-to-chemical conversion is of great promise for future energy demands, but often limited by the kinetically sluggish hydrogen evolution reaction (HER) on the surface of nonprecious transition metal catalysts in neutral media. It is thus highly desirable to rationally tailor the reaction interface to boost the neutral HER catalytic kinetics. Herein, we report a general surface nitrogen modification of diverse transition metals (e.g., iron, cobalt, nickel, copper, and nickel-cobalt alloy), accomplished by a facile low-temperature ammonium carbonate treatment, for significantly improved hydrogen generation from neutral water. Various physicochemical characterization techniques including synchrotron X-ray absorption spectroscopy (XAS) and theory modeling demonstrate that the surface nitrogen modification does not change the chemical composition of the underlying transition metals. Notably, the resulting nitrogen-modified nickel framework (N-Ni) exhibits an extremely low overpotential of $64 \mathrm{mV}$ at $10 \mathrm{~mA} \mathrm{~cm} \mathrm{~cm}^{-2}$, which is, to our knowledge, the best among those nonprecious electrocatalysts reported for hydrogen evolution at $\mathrm{pH}$ 7. Our combined experimental results and density functional theory (DFT) calculations reveal that the surface electron-rich nitrogen simultaneously facilitates the initial adsorption of water via the electron-deficient $\mathrm{H}$ atom and the subsequent dissociation of the electron-rich $\mathrm{HO}-\mathrm{H}$ bond via $\mathrm{H}$ transfer to $\mathrm{N}$ on the nickel surface, beneficial to the overall hydrogen evolution process.
\end{abstract}

\section{INTRODUCTION}

Natural photosynthesis that harnesses solar energy to convert $\mathrm{CO}_{2}$ and water to value-added chemical products and $\mathrm{O}_{2}$ is of paramount significance to mankind, ${ }^{1}$ albeit the overall energy conversion efficiency is rather mediocre. ${ }^{1,2}$ Therefore, considerable efforts have been devoted to developing artificial photosynthesis such as solar-driven water splitting cells ${ }^{3}$ and hybrid inorganic-biological systems, ${ }^{2,4}$ to mimic the nature's energy cycle with higher efficiency, which in turn would alleviate our dependence on fossil fuels. Considering the environmental impact and system cost as well as the biocompatibility with biocatalysts including bacteria (e.g., Methanosarcina barkeri, Ralstonia eutropha, and Moorella thermoacetica) and enzymes (e.g., hydrogenase and formate dehydrogenase), ${ }^{3}$ these assembled photosynthetic systems are preferred to function in neutral electrolytes and catalyzed by inexpensive transition metals or their compounds. ${ }^{2,3-7}$ For instance, Nocera's group has demonstrated a hybrid inorganic-biological system that employs Co-P as the $\mathrm{H}_{2}$ evolution catalyst in combination with Ralstonia eutropha to split water into $\mathrm{H}_{2}$ and $\mathrm{O}_{2}$, and in turn to synthesize biomass, fuels, and/or chemicals upon $\mathrm{CO}_{2}$ addition in neutral electrolyte (0.1 M KPi, pH 7). ${ }^{2}$ In addition, Chang and Yang et al. recently reported a hybrid bioinorganic approach for the solar-to-chemical conversion in $1 \mathrm{M}$ phosphate buffer $(\mathrm{pH} 7)$ wherein $\mathrm{H}_{2}$ generation was catalyzed by NiS and the produced $\mathrm{H}_{2}$ was then later taken up by living cells to convert $\mathrm{CO}_{2}$ to chemical fuels like $\mathrm{CH}_{4} .^{5}$ Leveraging these advances requires efficient and earth-abundant catalysts to further promote the kinetically sluggish $\mathrm{H}_{2}$ evolution reaction (HER) under neutral condition. ${ }^{6,8}$

Akin to many other electrocatalytic processes, HER takes place at the electrocatalyst/electrolyte interface and thus tuning the surface structure of the underlying catalysts would result in tailored and improved electrocatalytic performance. ${ }^{9-20}$ To date, most efforts focused on controlling particle size and shape, ${ }^{9,10}$ composition and defects, ${ }^{12-16}$ and creating the well-defined metal-support interface by using metal oxide/hydr(oxy)oxide support. ${ }^{17-20}$ For example, shaping the $\mathrm{Pt}_{3} \mathrm{Ni}$ polyhedrons with frame-like nanoarchitectures can boost their HER activity in alkaline solution. ${ }^{10}$ Doping $\mathrm{MoS}_{\mathrm{x}}$ with cobalt enhances the nanocatalyst's activity and stability for HER in both alkaline and acidic environments. ${ }^{14}$ Arranging the $\mathrm{Ni}(\mathrm{OH})_{2}$ nanoclusters on Pt surfaces can further promote the alkaline HER activity owing to their strong coupling interactions. ${ }^{17}$ In contrast, little attention has been paid on the surface heteroatom (such as nitrogen) modification of transition metals for accelerated HER under neutral condition.

Herein, we report a general surface nitrogen modification strategy to remarkably improve the neutral HER performance of diverse transition metals (e.g., iron, cobalt, nickel, copper, and nickel-cobalt alloy) through facile low-temperature ammonium carbonate treatment. Various physicochemical characterization techniques including synchrotron X-ray absorption spectroscopy (XAS) and theory modeling reveal that the surface 
nitrogen modification does not change the chemical composition of the underlying transition metals. However, significantly improved HER activities can be observed. Remarkably, the resulting surface nitrogen-modified porous nickel framework (N$\mathrm{Ni}$ ) only needs a very low overpotential of $64 \mathrm{mV}$ to achieve 10 $\mathrm{mA} \mathrm{cm}{ }^{-2}$, which makes N-Ni the best among those most active nonprecious HER electrocatalysts in neutral electrolyte. Moreover, on the basis of a series of experimental results and density functional theory (DFT) calculations, it is rationalized that the surface electron-rich nitrogen atoms not only favor the initial water adsorption but also facilitate the following dissociation of water on nickel surface, synergistically leading to the significant enhancement in HER activity at $\mathrm{pH} 7$.

\section{RESULTS AND DISCUSSION}

For the synthesis of surface nitrogen-modified nickel framework (N-Ni), porous nickel microsphere arrays were first grown on commercial nickel foam by template-free cathodic electrodeposition at a constant current density. Subsequently, the resulting nickel framework was subjected to lowtemperature ammonium carbonate treatment to obtain the $3 \mathrm{D}$ hierarchically porous N-Ni (see the Experimental Section for details). Low-magnification scanning electron microscopy (SEM) image reveals an interconnected, 3D macroporous network of N-Ni (Figure 1a), analogous to those of the pristine nickel foam and Ni framework (Figure S1a, c in Supporting Information). High-magnified SEM image of N-Ni exhibits an interesting structure composed of stacked nanoparticles (Figure 1b). This is in sharp contrast to the relatively flat surface observed for the nickel foam substrate (Figure S1b). A closer inspection of these nanoparticles in a high-resolution SEM (HR-SEM) image suggests the flocculent surface of $\mathrm{N}-\mathrm{Ni}$ (Figure 1b inset). No apparent differences in morphology are observed for $\mathrm{N}-\mathrm{Ni}$ and $\mathrm{Ni}$ framework (Figure S1d), which expedite direct comparison of their electrocatalytic HER activities. Fast Fourier transform (FFT) diffractogram of the region III (Figure 1c inset) in the transmission electron microscopy (TEM) image (Figure 1c) for N-Ni can be indexed to metallic nickel. ${ }^{21}$ HR-TEM images taken from different regions marked in Figure 1c clearly indicate the (111) plane of metallic nickel with a lattice fringe of $0.21 \mathrm{~nm}$ (Figure 1d), in accordance with the FFT result. The compositional linescanning profiles along the blue dash arrow in Figure 1c suggest the main composition of metallic nickel in N-Ni (Figure 1e). Moreover, the nearly identical XRD patterns of $\mathrm{N}-\mathrm{Ni}$ and $\mathrm{Ni}$ framework imply the inheritance of crystalline phase of $\mathrm{N}-\mathrm{Ni}$ upon ammonium carbonate treatment (Figure 1f), which is further confirmed by the following X-ray absorption spectroscopy (XAS) and X-ray photoelectron spectroscopy (XPS) measurements.

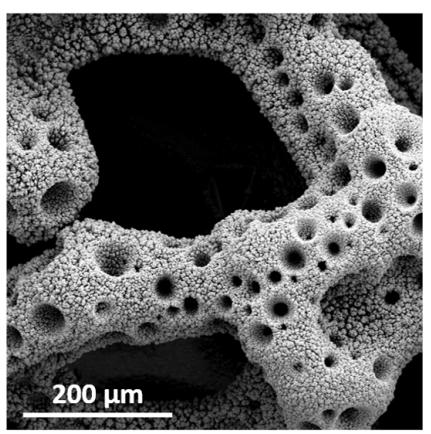

d

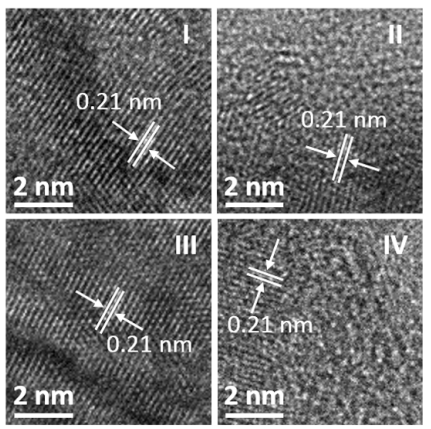

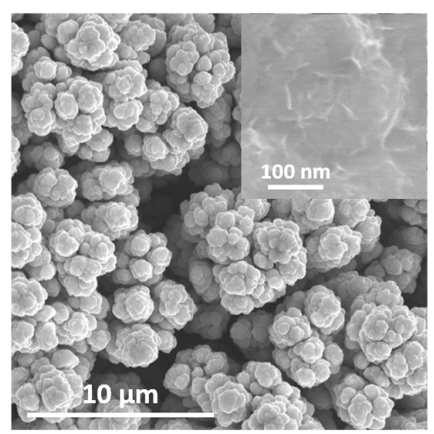

e

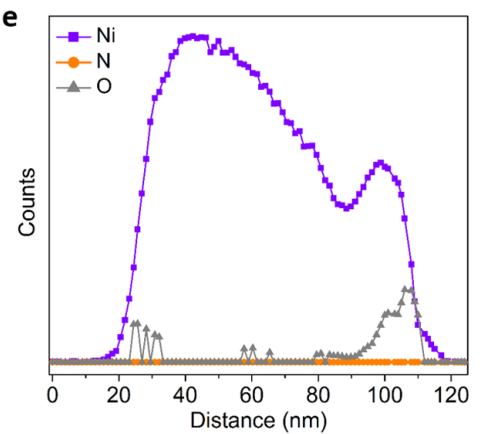

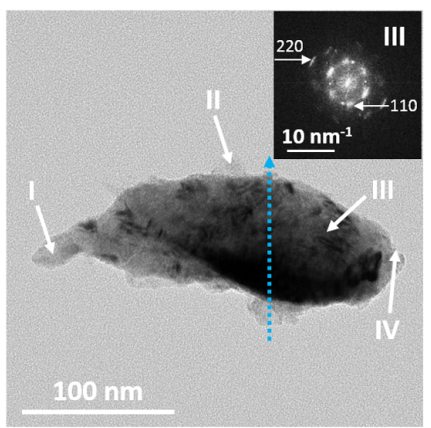

$\mathbf{f}$

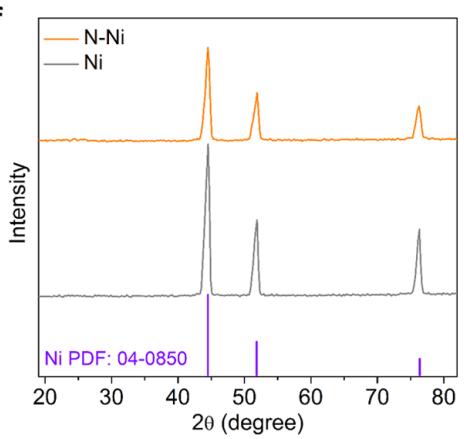

Figure 1. (a,b) SEM images of N-Ni at different magnifications. (c) TEM image of N-Ni. The inset shows the corresponding FFT diffractogram in region III. (d) HR-TEM images of N-Ni at different regions marked in (c). (e) Compositional line-scanning profiles along the blue dash arrow in (c). (f) XRD patterns of $\mathrm{N}-\mathrm{Ni}$ and $\mathrm{Ni}$ framework, along with the standard pattern of Ni. 

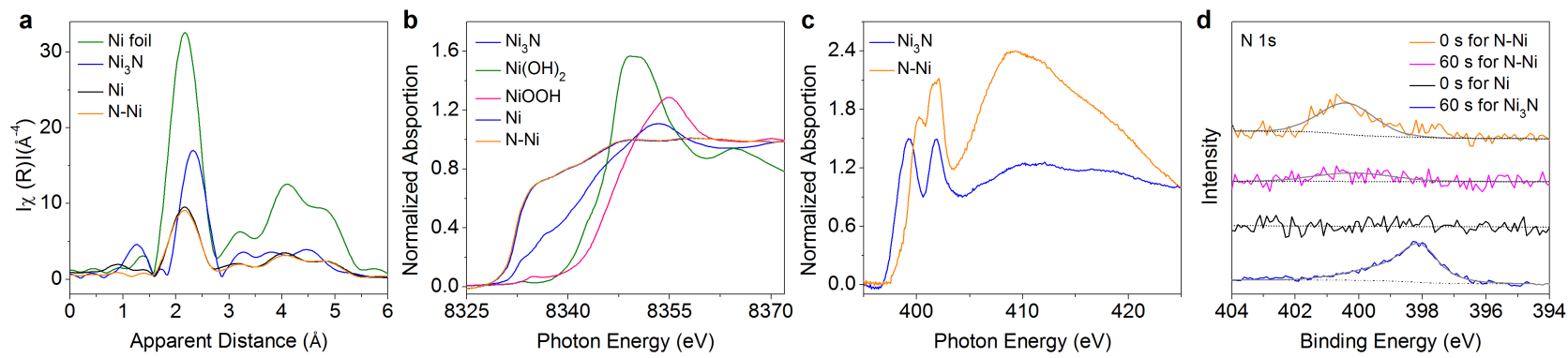

Figure 2. (a) Ni K-edge EXAFS spectra and (b) Ni K-edge XANES spectra of N-Ni and Ni framework, together with $\mathrm{Ni}$ foil, $\mathrm{Ni}{ }_{3} \mathrm{~N}, \mathrm{Ni}(\mathrm{OH})_{2}$, and NiOOH control samples. (c) N K-edge XANES spectra of N-Ni and Ni framework. (d) High-resolution N 1s spectra of N-Ni after Ar ${ }^{+}$ sputtering for 0 and $60 \mathrm{~s}$, together with that of fresh $\mathrm{Ni}$ framework and $\mathrm{Ni}_{3} \mathrm{~N}$ as control samples.

The X-ray absorption near-edge structure (XANES) and extended X-ray absorption fine structure (EXAFS) spectra of $\mathrm{N}-\mathrm{Ni}$ and $\mathrm{Ni}$ framework at the Ni K-edge region were collected (Figure 2a, b and S2). As shown in Figure $2 \mathrm{a}$ and S2, the Ni Kedge EXAFS spectra and FT $\kappa^{3}$-weighted oscillation curves of both N-Ni and Ni framework almost overlap, which are similar to those of the nickel foil benchmark but drastically different from those of $\mathrm{Ni}_{3} \mathrm{~N}$. Additionally, the corresponding XANES analysis further verifies the similarity between $\mathrm{N}-\mathrm{Ni}$ and $\mathrm{Ni}$ framework, and unambiguously excludes the involvement of $\mathrm{Ni}_{3} \mathrm{~N}, \mathrm{Ni}(\mathrm{OH})_{2}$, and $\mathrm{NiOOH}$ (Figure $2 \mathrm{~b}$ ). Furthormore, the $\mathrm{N}$ $\mathrm{K}$-edge XANES spectrum of N-Ni in comparison with that of $\mathrm{Ni}_{3} \mathrm{~N}$ positively shifts to higher energy (Figure $2 \mathrm{c}$ ), suggesting a much weaker interaction between $\mathrm{Ni}$ and $\mathrm{N}$ in $\mathrm{N}-\mathrm{Ni}$, which would cause less electron transfer from Ni to N. Since XAS data represents the bulk samples, XPS was also employed to investigate their surface compositions. Despite the close overlap of the high-resolution Ni $2 p$ XPS spectra of N-Ni and $\mathrm{Ni}$ framework (Figure S3), the high-resolution N 1s spectrum of N-Ni indeed indicates the presence of nitrogen (Figure 2d), in line with the $\mathrm{N}$ K-edge XANES results (Figure 2c). Even after N-Ni was subjected to argon sputtering for $60 \mathrm{~s}$, some nitrogen residues were still observed, in sharp contrast to the fresh Ni framework which only exhibited noise in the $\mathrm{N} 1 \mathrm{~s}$ region. Similar to the N K-edge XANES results (Figure 2c), the $\mathrm{N}$ 1s XPS peak of $\mathrm{N}-\mathrm{Ni}$ also positively shifts to higher binding energy relative to that of $\mathrm{Ni}_{3} \mathrm{~N}$, corroborating a much weaker interaction between $\mathrm{Ni}$ and $\mathrm{N}$ in $\mathrm{N}-\mathrm{Ni}$. Collectively, these characterization results unambiguously validate the successful surface nitrogen modification of $\mathrm{Ni}$ framework rather than the formation of nickel nitrides. We tentatively attribute this surface nitrogen modification to the facile decomposition of ammonium carbonate at relatively low temperature and the short interaction time of the released $\mathrm{NH}_{3}$ with the nickel microarrays deposited on the nickel foam, as elongated nitridation under $\mathrm{NH}_{3}$ would lead to nickel nitrides (Figure S4a). Similar formation of surface nitrogen adatoms on transition metals have been reported for nitrogen overlayers on iron. ${ }^{22}$

To glean deeper insights into the structural configuration of $\mathrm{N}-\mathrm{Ni}$ and compare it with $\mathrm{Ni}_{3} \mathrm{~N}$, DFT calculations were conducted. With one nitrogen adatom on a model nickel slab, the DFT-optimized structure (Figure 3a) indicates that $\mathrm{N}$ is prefered to located at the fcc hollow site of $\mathrm{Ni}$ and bound to three surface $\mathrm{Ni}$ atoms strongly. The distance between $\mathrm{N}$ and $\mathrm{Ni}$ for $\mathrm{N}-\mathrm{Ni}$ is $1.761 \AA$, smaller than that of $\mathrm{Ni}_{3} \mathrm{~N}(1.901 \AA$, Figure $3 \mathrm{~b})$. With Bader charge analysis, partial atomic charge on $\mathrm{N}$ in $\mathrm{N}-\mathrm{Ni}$ is found to be $-0.7459|\mathrm{e}|$, less negative than that of $\mathrm{Ni}_{3} \mathrm{~N}$ $(-0.912|\mathrm{e}|$, Figure $3 \mathrm{c})$. These calculations further confirm less electron transfer from $\mathrm{Ni}$ to $\mathrm{N}$ in $\mathrm{N}-\mathrm{Ni}$ than that in $\mathrm{Ni}_{3} \mathrm{~N}$, in agreement with the XPS and XAS results.
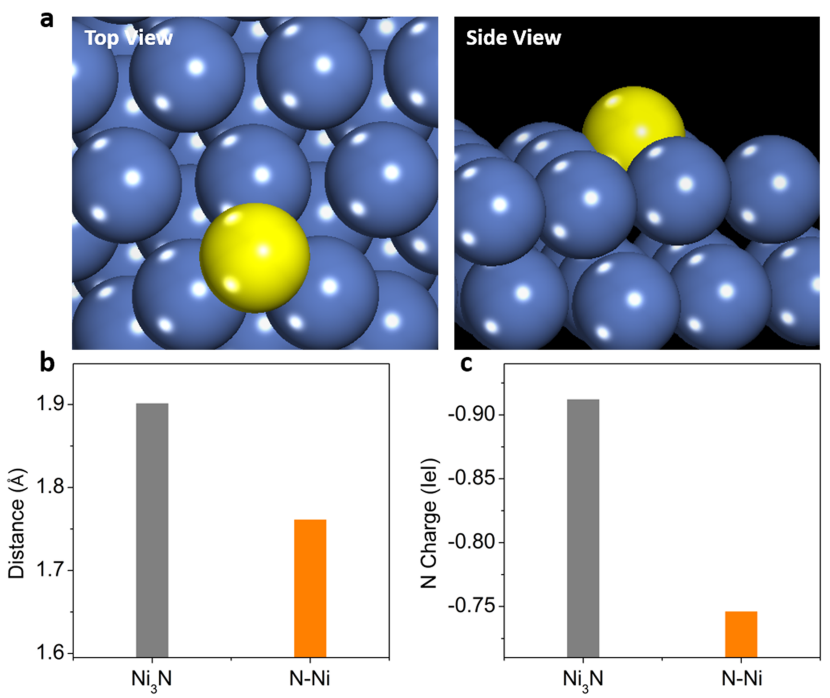

Figure 3. (a) DFT-optimized structure of N-Ni. Comparison of (b) the distance between $\mathrm{N}$ and $\mathrm{Ni}$ and (c) the partial atomic charge of $\mathrm{N}$ for $\mathrm{N}-\mathrm{Ni}$ and $\mathrm{Ni}_{3} \mathrm{~N}$.

Electrocatalytic HER measurements of $\mathrm{N}-\mathrm{Ni}$ and $\mathrm{Ni}$ framework were first studied by steady-state linear sweep voltammetry (LSV) in the neutral electrolyte (1.0 M phosphate buffer at $\mathrm{pH} 7$ ) with a three-electrode configuration. The Ni framework under a similar annealing procedure in argon gas (denoted as "Ni-a") and the state-of-the-art commercial $\mathrm{Pt} / \mathrm{C}$ $(20 \%)$ catalyst were also included for comparison. The observed LSV curve of N-Ni indicates a much smaller onset potential of nearly $0 \mathrm{mV}$ vs the reversible hydrogen electrode (RHE) and greater catalytic current density, compared to that of $\mathrm{Ni}$ framework (Figure 4a). In contrast, similar annealing process under argon instead of ammonium carbonate for the $\mathrm{Ni}$ a sample gives rise to almost identical HER activity as that of Ni framework (Figure 4a). These results distinctly highlight the important role of the low-temperature ammonium carbonate treatment for N-Ni. It's necessary to mention that all the three samples of N-Ni, Ni framework, and Ni-a share similar physical surface areas and mass loadings as they were all prepared following the same electropdepostion processess prior to subsequent treatment. Remarkably, our N-Ni only requires an overpotential of $64 \mathrm{mV}$ to reach the benchmark catalytic current density of $10 \mathrm{~mA} \mathrm{~cm}^{-2}$, which is much lower than that of $\mathrm{Ni}$ framework, Ni-a and most recently reported nonprecious HER 
catalysts at $\mathrm{pH} 7$, including $\mathrm{Co}-\mathrm{P}(>137 \mathrm{mV}),{ }^{2} \mathrm{NiS}(\sim 387$ $\mathrm{mV}),{ }^{5}$ amorphous $\mathrm{MoS}_{\mathrm{x}}(>290 \mathrm{mV}),{ }^{23} \mathrm{NiMoZn}$ film $(\sim 187$ $\mathrm{mV}),{ }^{24} \mathrm{H}_{2}$-CoCat $(>500 \mathrm{mV}),{ }^{25} \mathrm{Ni}-\mathrm{Mo}-\mathrm{S} / \mathrm{C}(200 \mathrm{mV}),{ }^{26}$ and
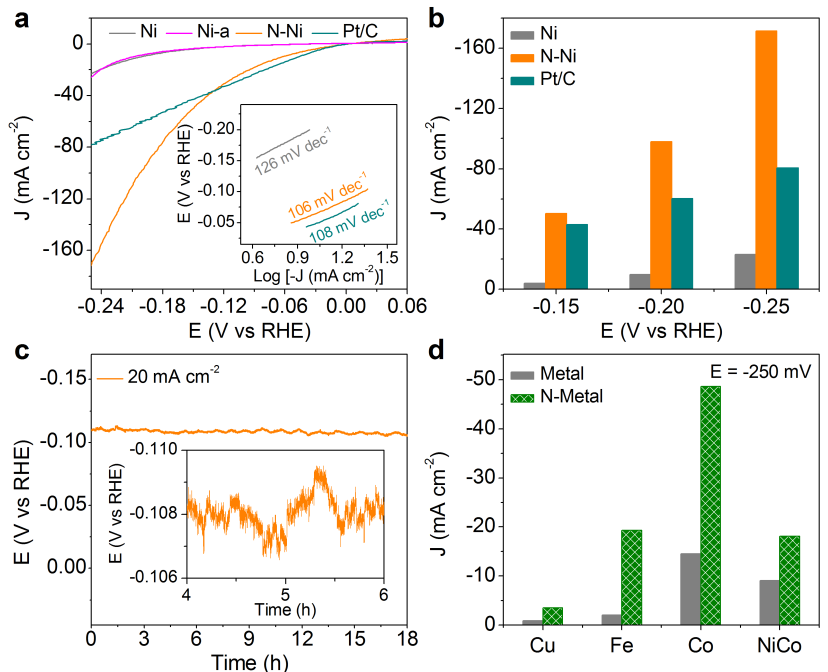

Figure 4. (a) LSV curves of N-Ni, Ni framework, Ni-a, and $\mathrm{Pt} / \mathrm{C}$ in $1.0 \mathrm{M} \mathrm{pH}=7$ phosphate buffer. The inset shows the corresponding Tafel plots. (b) Comparison of the current densities at different overpotentials for N-Ni, Ni framework, and Pt/C. (c) Chronopotentiometric curve of $\mathrm{N}-\mathrm{Ni}$ at $20 \mathrm{~mA} \mathrm{~cm}{ }^{-2}$. The inset shows the expanded chronopotentiometric curve with oscillations due to the growth and release of $\mathrm{H}_{2}$ bubbles on $\mathrm{N}-\mathrm{Ni}$ surface. (d) Comparison of the current densities at the overpotential of $250 \mathrm{mV}$ for porous $\mathrm{Cu}, \mathrm{Fe}, \mathrm{Co}$ and $\mathrm{NiCo}$ alloy samples before (Metal) and after $(\mathrm{N}-$ Metal) surface nitrogen modification.

$\mathrm{h}^{-\mathrm{NiS}_{\mathrm{x}}}(210 \mathrm{mV}) .{ }^{27}$ In addition, N-Ni achieves 49.5, 97.1, and $170 \mathrm{~mA} \mathrm{~cm}^{-2}$ at overpotentials of 150,200 , and $250 \mathrm{mV}$, which are 7.4 12.6 times higher than those of Ni framework and also 1.16 2.13 times better than those of Pt/C (Figure 4b). A more detailed comparison of the neutral HER activity is included in Table S1. To the best of our knowledge, such a superior HER activity of a nonprecious catalyst in neutral media has not been reported. The substantially enhanced HER activity of N-Ni in comparison to that of $\mathrm{Ni}$ framework is also corroborated by its smaller semicircular diameter in the electrochemical impedance spectrum (Figure S5), implying smaller charge transfer resistance of the former. Note that the HER activity of our N$\mathrm{Ni}$ is also much higher than that of nickel nitride supported on nickel foam $\left(\mathrm{Ni}_{3} \mathrm{~N} / \mathrm{Ni}\right.$, Figure $\mathrm{S} 4 \mathrm{~b}$ ), confirming the successful surface nitrogen modification of $\mathrm{Ni}$ framework and then indirectly excluding the formation of nickel nitrides. These results manifest that the HER kinetics is sensitive to the surface structure of catalysts in neutral media, consistent with previous report. ${ }^{17}$ Other than high activity, our N-Ni also exhibits excellent long-term electrochemical robustness, as evidenced by its stable overpotential of $109 \mathrm{mV}$ to reach $20 \mathrm{~mA} \mathrm{~cm}^{-2}$ for an $18 \mathrm{~h}$ chronopotentiometry experiment (Figure 4c). The produced $\mathrm{H}_{2}$ was quantified via gas chromatography and a nearly unity Faradaic efficiency was obtained. The fluctuations in an expanded chronopotentiometric curve also implies the formation and release of $\mathrm{H}_{2}$ bubbles on the $\mathrm{N}-\mathrm{Ni}$ catalyst surface (Figure 4c inset).

In order to further explore the generality of this surface nitrogen engineering, other transition metals and alloys such as porous iron, cobalt, copper, and nickel-cobalt alloy were prepared and subjected to the same ammonium carbonate treatment.
SEM, XRD and XPS characterizations (Figure S6-S9) suggest that all the resulting nitrogen-modified transition metal $(\mathrm{N}$ Metal) samples maintain the surface nitrogen existence, porous morphology, and corresponding metal crystalline phases, similar to N-Ni. More importantly, all the measured LSV curves of these N-Metal samples show that the catalytic currents are shifted to significantly lower overpotentials compared to those of the parent metals at $\mathrm{pH} \mathrm{7,} \mathrm{indicative} \mathrm{of} \mathrm{the} \mathrm{enhanced} \mathrm{HER}$ activities after ammonium carbonate treatment (Figure S10). For example, the current densities of N-Fe, N-Co, N-Cu, and N$\mathrm{NiCo}$ at an overpotential of $250 \mathrm{mV}$ rise from 2.05, 14.6, 0.93, and 9.1 to $19.3,48.8,3.4$, and $18.1 \mathrm{~mA} \mathrm{~cm}^{-2}$, respectively (Figure 4d), strongly proving the versatility of our surface nitrogen modification in improving the HER activities of inexpensive transition metal catalysts in neutral media.

The generally accepted reaction mechanism of HER in neutral and alkaline solution follows either the Volmer-Heyrosky or the Volmer-Tafel step, where the initial electrochemical water adsorption and subsequent water dissociation (parts of Volmer step) are considered as the rate-limiting step and result in a theoretical Tafel slope of $118 \mathrm{mV} \mathrm{dec}^{-1}{ }^{5,28,29}$ The calculated Tafel slopes for both $\mathrm{N}-\mathrm{Ni}$ and $\mathrm{Ni}$ framework, along with $\mathrm{Pt} / \mathrm{C}$, are quite close to the theoretical prediction (Figure $4 \mathrm{a}$ inset), implying the critical role of Volmer step for HER at $\mathrm{pH}$ 7. Based on the above electrocatalytic investigation and Tafel slope analysis, we envision that the surface nitrogen modification may facilitate the initial water adsorption and/or the following water dissociation on the surface of $\mathrm{N}-\mathrm{Ni}$.

To understand the improved HER activity of N-Ni after surface nitrogen modification, we performed DFT calculations to determine the difference in water adsorption and dissociation between $\mathrm{Ni}$ framework and N-Ni. As argued by Norskov et $a l .,{ }^{30}$ hydrogen adsorption can be used as a powerful descriptor for predicting many catalysts' HER activities under acidic conditions. We reason that examining the adsorption and dissociation of a water molecule on the catalyst surface can provide very useful insights into the qualitative trend of HER activity under the neutral condition wherein our catalysts were tested. We modeled the nickel catalyst by the lowest-energy surface of bulk nickel, Ni(111). For the structure of N-Ni, we assumed that a very reasonable model was $\mathrm{N}$-adatom-modified $\mathrm{Ni}(111)$ based on DFT calculations. We found that $\mathrm{N}$ prefers the fcc-hollow site on Ni(111) (Figure 3a). As discussed above, the low interaction of the initial water adsorption and the high kinetic energy barrier of the subsequent water dissociation on the surface of nickel are responsible for the sluggish HER kinetics in neutral solution. Accordingly, an ideal catalyst should have sufficient affinity to bind water to accelerate the initial electron-transfer process but also possess enough repellency to facilitate the following water dissociation. ${ }^{31}$ On the clean Ni(111) surface (Figure 5a and S11a), water adsorbs atop a surface Ni atom. Interestingly, water adsorption is enhanced by over $0.11 \mathrm{eV}$ on $\mathrm{N}$ $\mathrm{Ni}(111)$ where the surface nitrogen atom forms a hydrogen bond with $\mathrm{H}-\mathrm{OH}$ through the electron-deficient $\mathrm{H}$ atom in $\mathrm{H}_{2} \mathrm{O}$ (Figure $5 \mathrm{~b}$ and $\mathrm{S} 1 \mathrm{~b}$ ), leading to a change in adsorption energy ( $\left.E_{\text {ads }}\right)$ from $-0.30 \mathrm{eV}$ for $\mathrm{Ni}$ to $-0.41 \mathrm{eV}$ for N-Ni (Figure $5 \mathrm{c}$ ). Subsequently, the minimum-energy paths for water dissociation on both $\mathrm{Ni}$ and N-Ni were calculated (Figure 5d, e and S12). The energy barrier for water dissociation on the N-Ni slab is found to be $0.53 \mathrm{eV}$, significantly lower than that on $\mathrm{Ni}(0.96$ $\mathrm{eV}$, Figure $5 \mathrm{~d}$, e and S13). If we take into account of the zeropoint-energy and entropic corrections, the free-energy barriers 
lower to $0.42 \mathrm{eV}$ on $\mathrm{N}-\mathrm{Ni}(111)$ and $0.84 \mathrm{eV}$ on $\mathrm{Ni}(111)$, as shown in Figure 5f.
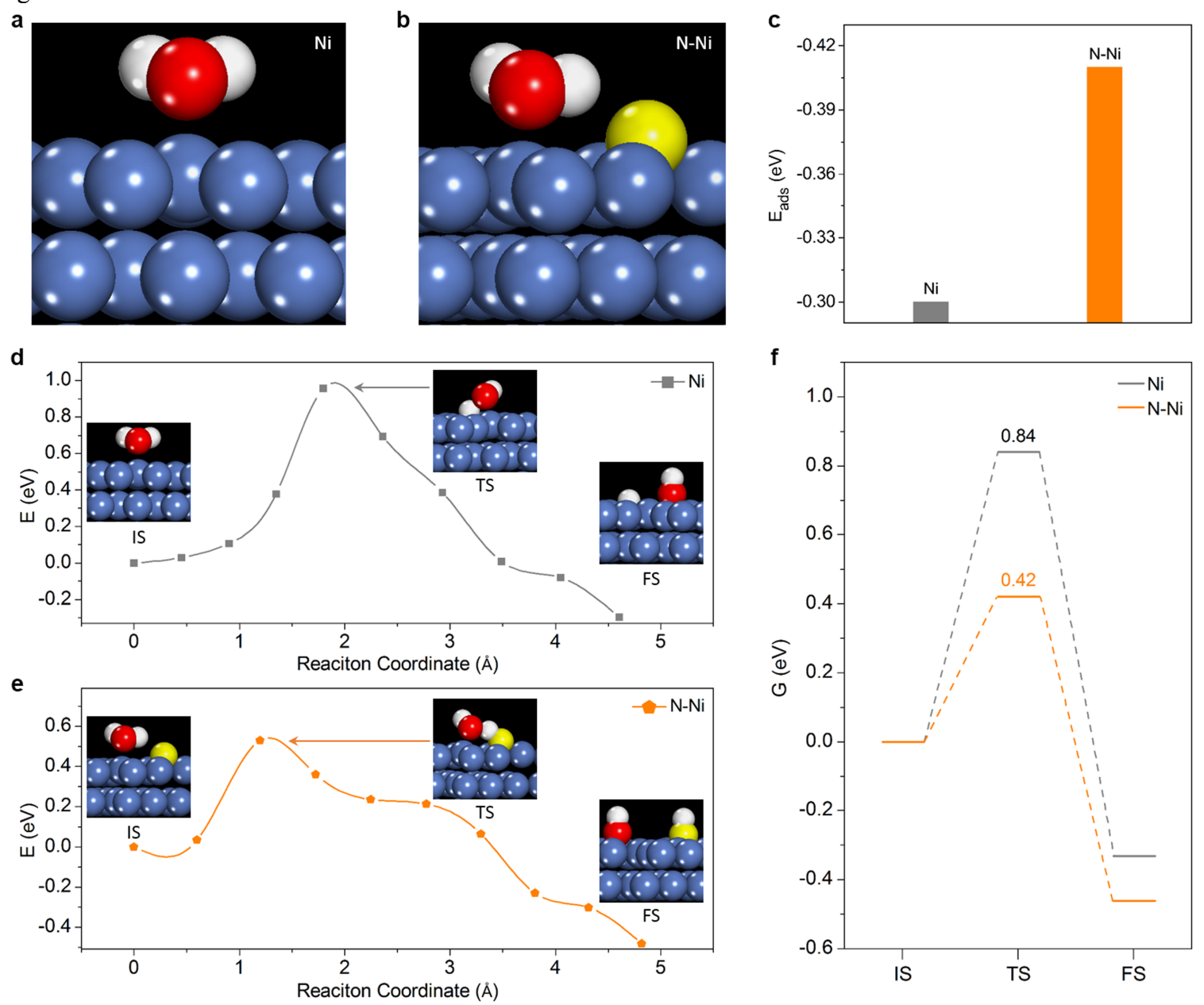

Figure 5. DFT calculations of water adsorption and dissociation on $\mathrm{Ni}(111)$ and $\mathrm{N}-\mathrm{Ni}(111)$ : (a) Optimized structure of water adsorption on $\mathrm{Ni}(111)$. (b) Optimized structure of water adsorption on N-Ni(111). (c) Adsorption energy of water on Ni(111) and N-Ni(111). Minimumenergy paths for water dissociation on (d) Ni(111) and (e) N-Ni(111) with structures for initial (IS), transition (TS), and final (FS) states. (f) Comparison of free-energy barrier profiles of water dissociation on $\mathrm{Ni}(111)$ and $\mathrm{N}-\mathrm{Ni}(111)$, taking into account of the zero-point-energy and entropic corrections. Color code: blue, Ni; yellow, N; red, O; white, $\mathrm{H}$.

In other words, the surface $\mathrm{N}$ atom greatly facilitates water dissociation. From the structures of the transition states (Figure $5 \mathrm{~d}$ ), one can see that the water molecule has to bend significantly toward the surface for $\mathrm{HO}-\mathrm{H}$ bond to break on $\mathrm{Ni}(111)$, while the $\mathrm{H}$ atom can be facilely transferred to $\mathrm{N}$ along the already formed HO-H---N hydrogen bond on $\mathrm{N}-\mathrm{Ni}(111)$ (Figure 5e). Although fully considering the liquid environment and the applied potentials in DFT modeling is much more challenging and we have only explored the initial steps of HER, the present DFT results have clearly illustrated that the surface nitrogen modification achieved by ammonium carbonate treatment not only facilitates the initial water adsorption but also expedites the subsequent water dissociation, cooperatively resulting in the significant promotion of HER activity for N-Ni under neutral condition. Our DFT results also suggest that one can think of the HER active site of N-Ni(111) as an ensemble of the N-adatom and the nearby $\mathrm{Ni}$ atoms around it.

It is putative that the mechanism of HER in alkaline media resembles that in neutral solution. Therefore, a similar activity improvement from surface nitrogen modification is anticipated for HER in alkaline media. ${ }^{32}$ Indeed, the LSV curves of diverse transition metals frameworks collected in $1.0 \mathrm{M} \mathrm{KOH}$ (Figure
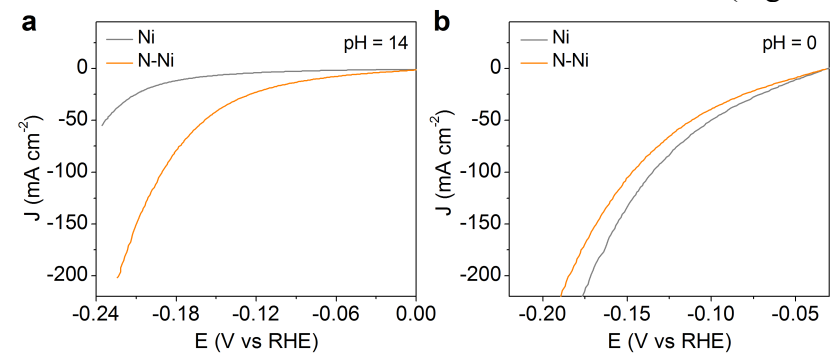

Figure 6. LSV curves of N-Ni and Ni framework in (a) alkaline (1.0 M KOH, $\mathrm{pH}=14)$ and (b) acidic $\left(0.5 \mathrm{M} \mathrm{H}_{2} \mathrm{SO}_{4}, \mathrm{pH}=0\right)$ solutions.

6a and S14) clearly demonstrate the much enhanced HER activities after similar ammonium carbonate treatment. However, under acidic condition, the adsorption of hydrogen is widely used as a descriptor to assess the HER performance of various 
catalysts. ${ }^{30}$ It is shown that an adsorption free energy of hydrogen $\left(\Delta \mathrm{G}_{\mathrm{H}}\right)$ close to zero usually results in high performance for $\mathrm{H}_{2}$ evolution at $\mathrm{pH} 0 .{ }^{33}$ Based on our DFT calculations, it is found that the nitrogen atom in N-Ni leads to a stronger interaction of proton on N-Ni than on Ni framework (Figure S15), which is disadvantageous to HER under acidic condition. Therefore, rather than improved but decreased HER activity would be expected for $\mathrm{N}-\mathrm{Ni}$ compared to $\mathrm{Ni}$ framework at $\mathrm{pH}$ 0 . This hypothesis is well confirmed by the LSV results of N$\mathrm{Ni}$ and $\mathrm{Ni}$ framework collected in the $0.5 \mathrm{M} \mathrm{H}_{2} \mathrm{SO}_{4}$ electrolyte (Figure 6b). Note that both $\mathrm{N}-\mathrm{Ni}$ and $\mathrm{Ni}$ catalysts are not very stable in acidic media, so that the initial LSV curves for both were recorded for comparison. These control electrochemical measurements (Figure 6 and S14) further substantiate our DFT prediction experimentally and complement our studies at $\mathrm{pH} 7$ and 14. Hence, although $\Delta \mathrm{G}_{\mathrm{H}}$ is a good descriptor for HER at $\mathrm{pH} 0$, our DFT results and experimental LSV curves show that it may not apply to HER under neutral and alkaline conditions. Instead, we have shown that water adsorption and dissociation could be key factors dictating HER activity at $\mathrm{pH} 7$.

\section{CONCLUSIONS}

In summary, we have presented a general strategy of surface nitrogen modification for diverse transition metals (e.g., iron, cobalt, nickel, copper and nickel-cobalt alloy) to significantly promote their hydrogen evolution performance in neutral media. For instance, upon ammonium carbonate treatment at relatively low temperature, nickel framework maintained its overall morphology and the main crystalline phase was still metallic nickel, as confirmed by various physicochemical characterization techniques and DFT calculations. Remarkably, the resulting surface nitrogen-modified porous nickel framework exhibited unprecedented electrocatalytic activity for hydrogen evolution at $\mathrm{pH} \mathrm{7,} \mathrm{requiring} \mathrm{a} \mathrm{low} \mathrm{overpotential} \mathrm{of} \mathrm{only} 64 \mathrm{mV}$ to produce $10 \mathrm{~mA} \mathrm{~cm}^{-2}$. To the best of our knowledge, it represents the most active catalyst among all the recently reported nonprecious HER electrocatalysts in neutral electrolyte. DFT computations were further utilized to understand the beneficial role that the surface nitrogen plays in boosting the HER performance. It was found that the presence of nitrogen facilitates not only water adsorption but also water dissociation, both of which are critical steps for hydrogen evolution at $\mathrm{pH}$ 7. In addition, surface nitrogen was theoretically predicted to be beneficial for HER under basic condition but disadvantageous under acidic condition. This hypothesis is also well validated by our experimental results. Overall, our work documents an effective and facile approach to substantially boost the HER activities of inexpensive metal catalysts under neutral and alkaline conditions by atomic-level surface engineering. The working principles, revealed by theoretical insights, can potentially lead to more competent electrocatalysts for hydrogen evolution under diverse conditions, especially for those applications in artificial photosynthesis.

\section{EXPERIMENTAL SECTION}

Synthesis of Surface N-Modified Transition Metals. All chemicals were used as received without any further purification. Commercial $\mathrm{Pt} / \mathrm{C}$ catalyst $(20 \% \mathrm{Pt}$ on Vulcan XC72) was purchased from Premetek. Deionized water $(18 \mathrm{M} \Omega \cdot \mathrm{cm})$ was used in all experiments. The N-Ni catalyst was prepared by a facile template-free cathodic electrodeposition of porous nickel microsphere arrays on nickel foam followed by lowtemperature ammonium carbonate treatment. Typically, the electrodeposition of 3D porous nickel microspheres on nickel foam (Ni framework) was performed in a standard twoelectrode glass cell at room temperature with an electrolyte consisting of $2.0 \mathrm{M} \mathrm{NH}{ }_{4} \mathrm{Cl}$ and $0.1 \mathrm{M} \mathrm{NiCl}_{2}$. A piece of commercial nickel foam with a size of $0.5 \mathrm{~cm} \times 0.5 \mathrm{~cm}$ was used as the working electrode and a Pt wire as the counter electrode. The electrodeposition was carried out at a constant current of $-1.0 \mathrm{~A} \mathrm{~cm}^{-2}$ for $500 \mathrm{~s}$ to obtain the Ni framework. Subsequently, the resulting Ni framework was placed at the center of a tube furnace, and $4.2 \mathrm{~g}$ ammonium carbonate was placed at the upstream side of the furnace at a carefully adjusted location. After flushed with Ar gas for $30 \mathrm{~min}$, the center of the furnace was quickly elevated to the reaction temperature of $420^{\circ} \mathrm{C}$ for $30 \mathrm{~min}$. After cooling down to room temperature, the product was washed with a large amount of water and ethanol, and finally the surface nitrogen-modified $\mathrm{Ni}$ framework $(\mathrm{N}-\mathrm{Ni})$ was obtained. A similar procedure was used to synthesize other surface nitrogen-modified transition metals (N-Metals). $0.1 \mathrm{M}$ $\mathrm{FeCl}_{2}, 0.1 \mathrm{M} \mathrm{CuSO}_{4}, 0.1 \mathrm{M} \mathrm{CoCl}_{2}$, and a mixture of $0.05 \mathrm{M}$ $\mathrm{NiCl}_{2}$ and $0.05 \mathrm{M} \mathrm{CoCl}_{2}$ instead of $0.1 \mathrm{M} \mathrm{NiCl}_{2}$ were used for the electrodeposition of the corresponding porous metals frameworks at $-1.0 \mathrm{~A} \mathrm{~cm}^{-2}$ for $200,15,200$ and $200 \mathrm{~s}$, respectively. A copper foam was used as a substrate for the preparation of $\mathrm{N}-\mathrm{Cu}$, and titanium foils were used as substrates for the preparation of $\mathrm{N}-\mathrm{Fe}, \mathrm{N}-\mathrm{Co}$ and $\mathrm{N}-\mathrm{NiCo}$ alloy, respectively. For the synthesis of $\mathrm{N}-\mathrm{Fe}$, the stock soluiton was firstly acidified to $\mathrm{pH} 2$ with $\mathrm{HCl}$.

Physical Methods. Scanning electron microscopy (SEM) and transmission electron microscopy (TEM) measurements were collected on a FEI QUANTA FEG 650 (FEI, USA) and a JEM-2800 (JEOL, Japan), respectively. X-ray diffraction (XRD) patterns were obtained on a Rigaku MinifexII Desktop X-ray diffractometer. The X-ray photoelectron spectroscopy analyses were performed using a Kratos Axis Ultra instrument (Chestnut Ridge, NY) at the Surface Analysis Laboratory, University of Utah Nanofab. The samples were affixed on a stainless steel Kratos sample bar, loaded into the instrument's load lock chamber, and also sputter cleaned inside the analysis chamber with $1 \mathrm{keV} \mathrm{Ar}{ }^{+}$ions for 0 and 60 seconds. X-ray absorption spectra (XAS) were collected at the Stanford Synchrotron Radiation Lightsource (SSRL) on beamline 9-3 with an electron energy of $3.0 \mathrm{GeV}$ and an average current of $500 \mathrm{~mA}$. This beamline uses a cryogenically cooled Si (220) double-crystal monochromator which was detuned to $50 \%$ of flux maximum at Ni K-edge. The incident and transmitted X-ray intensities were monitored by $\mathrm{N}_{2}$-filled ion chambers $\left(I_{0}\right.$, in front of the sample and $I_{l}$ after the sample). Absorption spectra were recorded in transmission mode (using ion chamber I1) as well as fluorescence excitation spectra using a 100-element Ge monolithic solid-state detector (Canberra). The monochromator energy was calibrated with $\mathrm{Ni}$ foil rising edge energy (8333.0 $\mathrm{eV})$. Boron nitride was used to dilute the samples $(\sim 1 \% \mathrm{w} / \mathrm{w})$ which were then packed into $0.5 \mathrm{~mm}$ thick aluminum sample holders using kapton film windows on both sides. Data reduction of the XAS spectra was performed using SamView (SixPack software, http://www.sams-xrays.com/sixpack). Preedge and post-edge backgrounds were subtracted from the absorption spectra using Athena software (IFEFFIT package), ${ }^{34}$ and resulting spectra were normalized with respect to the edge jump. A five-domain cubic spline was used for background removal in k-space. The extracted k-space data, $\mathrm{k}_{\chi}^{3}(\mathrm{k})$, was then Fourier transformed into r-space using a k-space window of $2.75-11.30 \AA^{-1}$. 
Electrocatalytic Measurements. Electrochemical measurements were performed by a computer-controlled Gamry Interface 1000 electrochemical workstation with a three-electrode cell system. The resulting catalysts were used as the working electrode, a $\mathrm{Ag} / \mathrm{AgCl}$ (sat. $\mathrm{KCl}$ ) electrode as the reference electrode, and a $\mathrm{Pt}$ wire as the counter electrode. When $\mathrm{Pt} / \mathrm{C}$ was utilized as the catalyst, its loading amount was $0.5 \mathrm{mg} \mathrm{cm}^{-2}$ on a Ni foam. All potentials reported herein were quoted with respect to reversible hydrogen electrode (RHE) through RHE calibration. iR (current times internal resistance) compensation was applied in all the electrochemical experiments to account for the voltage drop between the reference and working electrodes using Gamry Framework ${ }^{\mathrm{TM}}$ Data Acquisition Software 6.11 .

Theoretical Computation Methods. Spin-polarized density functional theory (DFT) calculations were performed using the Vienna ab initio simulation package (VASP). ${ }^{36}$ The ion-electron interaction was described with the projector augmented wave (PAW) method. ${ }^{36}$ Electron exchange-correlation was represented by the functional of Perdew, Burke and Ernzerhof (PBE) of generalized gradient approximation (GGA). ${ }^{37}$ A cutoff energy of $400 \mathrm{eV}$ was used for the plane-wave basis set. $\mathrm{Ni}(111)$ surface was modeled with five layers of slab in $(4 \times 4)$ lateral cells with $15 \AA$ of vacuum along the z-direction. The Brillouin zone was sampled by $(3 \times 3 \times 1)$ Monkhorst-Pack k-point mesh. The top two layers of the slab were allowed to relax together with the adsorbates and the convergence threshold for structural optimization was set to be $0.025 \mathrm{eV} \AA^{-1}$ in force. The climbingimage nudged elastic band (CI-NEB) method $^{38}$ implemented in VASP was used to determine the energy barriers of water splitting on $\mathrm{Ni}$ (111) and $\mathrm{N}-\mathrm{Ni}(111)$. The transition states were obtained by relaxing the force below $0.05 \mathrm{eV} \AA^{-1}$. The adsorption energies of water on $\mathrm{Ni}(111)$ and $\mathrm{N}-\mathrm{Ni}(111)$ were calculated by $E_{a d s}=E_{\text {water }+ \text { slab }}-E_{\text {slab }}-E_{\text {water }}$, where $E_{\text {water }+ \text { slab }}$, $E_{\text {slab }}$, and $E_{\text {water }}$ represent the total energy of the water-slab system, the total energy of the slab, and the energy of one gas phase water molecule, respectively. In addition, the adsorption energies of $\mathrm{H}$ on $\mathrm{Ni}(111)$ and $\mathrm{N}-\mathrm{Ni}(111)$ were calculated by $E_{a d s}=E_{H+s l a b}-E_{\text {slab }}-\frac{1}{2} E_{H_{2}}$, where $E_{H+\text { slab }}$ and $E_{H_{2}}$ represent the total energy of the $\mathrm{H}$-slab system and the energy of one gas phase hydrogen molecule, respectively. The transition states were verified to be rank-1 saddle point, i.e., having only one imaginary frequency. Zero-point-energy and entropic corrections were taken into account to assess free-energy barriers.

\section{ASSOCIATED CONTENT}

Supporting Information. Additional SEM images, electrochemical plots, EIS, XAS, XPS, XRD, EDX, DFT calculations and tables. This material is available free of charge via the Internet at http://pubs.acs.org.

\section{AUTHOR INFORMATION}

\section{Corresponding Author}

*djiang@ucr.edu

*yujie.sun@usu.edu.

\section{Author Contributions}

'B.Y., X.L., and G.H. contributed equally.

\section{Notes}

The authors declare no competing financial interests.

\section{ACKNOWLEDGMENT}

Y.S. acknowledges the financial support of Utah State University (USU) and the National Science Foundation (CHE1653978). Y.S. thanks the support of the Ralph E. Powe Junior Faculty Enhancement Award (ORAU) and the Microscopy Core Facility at USU. D.J. is supported by the University of California, Riverside. J.Y. is supported by the Director, Office of Science, Office of Basic Energy Sciences (OBES), Division of Chemical Sciences, Geosciences, and Biosciences of the Department of Energy (DOE) under Contract DE-AC0205CH11231. XAS data was collected at the Stanford Synchrotron Radiation Light source (SSRL) beamline 9-3, operated by Stanford University for the U.S. DOE Office of Science, and supported by the DOE Office of Biological and Environmental Research, and by the NIH (including P41GM103393). This research used resources of the National Energy Research Scientific Computing Center, a DOE Office of Science User Facility supported by the Office of Science of the U.S. Department of Energy under Contract No. DE-AC02-05CH11231.

\section{REFERENCES}

(1) Blankenship, R. E.; Tiede, D. M.; Barber, J.; Brudvig, G. W.; Fleming, G.; Ghirardi, M.; Gunner, M. R.; Junge, W.; Kramer, D. M.; Melis, A.; Moore, T. A.; Moser, C. C.; Nocera, D. G.; Nozik, A. J.; Ort, D. R.; Parson, W. W.; Prince, R. C.; Sayre, R. T. Science 2011, 332, 805-809.

(2) Liu, C.; Colón, B. C.; Ziesack, M.; Silver, P. A.; Nocera, D. G. Science 2016, 352, 1210-1213.

(3) Reece, S. Y.; Hamel, J. A.; Sung, K.; Jarvi, T. D.; Esswein, A. J.; Pijpers, J. J. H.; Nocera, D. G. Science 2011, 334, 645-648.

(4) Sakimoto, K. K.; Wong, A. B.; Yang, P. Science 2016, 351, 74 77.

(5) Nichols, E. M.; Gallagher, J. J.; Liu, C.; Su, Y.; Resasco, J.; Yu, Y.; Sun, Y.; Yang, P.; Chang, M. C. Y.; Chang, C. J. Proc. Natl. Acad. Sci. U. S. A. 2015, 112, 11461-11466.

(6) Kornienko, N.; Sakimoto, K. K.; Herlihy, D. M.; Nguyen, S. C.; Alivisatos, A. P.; Harris, C. B.; Schwartzberg, A.; Yang, P. Proc. Natl. Acad. Sci. U.S. A. 2016, 113, 11750-11755.

(7) Yadav, R. K.; Baeg, J. O.; Oh, G. H.; Park, N. J.; Kong, K.; Kim, J.; Hwang, D. W.; Biswas, S. K. J. Am. Chem. Soc. 2012, 134, 11455-11461.

(8) Sun, Y.; Liu, C.; Grauer, D. C.; Yano, J.; Long, J. R.; Yang, P.; Chang, C. J. J. Am. Chem. Soc. 2013, 135, 17699-17702.

(9) Schweinberger, F. F.; Berr, M. J.; Döblinger, M.; Wolff, C.; Sanwald, K. E.; Crampton, A. S.; Ridge, C. J.; Jäckel, F.; Feldmann, J.; Tschurl, M.; Heiz, U. J. Am. Chem. Soc. 2013, 135, 1326213265 .

(10) Chen, C.; Kang, Y.; Huo, Z.; Zhu, Z.; Huang, W.; Xin, H. L.; Snyder, J. D.; Li, D.; Herron, J. A.; Mavrikakis, M.; Chi, M.; More, K. L.; Li, Y.; Markovic, N. M.; Somorjai, G. A.; Yang, P.; Stamenkovic, V. R. Science 2014, 343, 1339-1343.

(11) Cummins, D. R.; Martinez, J.; Sherehiy, A.; Kappera, R.; Martinez-Garcia, A.; Schulze, R. K.; Jasinski, J.; Zhang, J.; Gupta, R. K.; Lou, J.; Chhowalla, M.; Sumanasekera, G.; Mohite, A. D.; Sunkara, M. K. Gupta, G. Nat. Commun. 2016, 7, 11857.

(12) Cabán-Acevedo, M.; Stone, M. L.; Schmidt, J. R.; Thomas, J. G.; Ding, Q.; Chang, H. C.; Tsai, M. L. Jin, S. Nat. Mater. 2015, 14, 1245-1251.

(13) Kibsgaard, J.; Jaramillo, T. J. Angew. Chem. Int. Ed. 2014, 53, 14433-14437.

(14) Staszak-Jirkovský, J.; Malliakas, C. D.; Lopes, P. P.; Danilovic, N.; Kota, S. S.; Chang, K. C.; Genorio, B.; Strmenik, D.; Stamenkovic, V. R.; Kanatzidis, M. G.; Markovic, N. M. Nat. Mater. 2016, 15, 197-203.

(15) Li, Y. H.; Liu, P. F.; Pan, L. F.; Wang, H. F.; Yang, Z. Z.; Zheng, L. R.; Hu, P.; Zhao, H. J. Gu, L.; Yang, H. G. Nat. Commun. 2015, 6, 8064 . 
(16) Yin, Y.; Han, J.; Zhang, Y.; Zhang, X.; Xu, P.; Yuan, Q.; Samad, L.; Wang, X.; Wang, Y.; Zhang, Z.; Zhang, P.; Cao, X.; Song, B.; Jin, S. J. Am. Chem. Soc. 2016, 138, 7965-7972.

(17) Subbaraman, R.; Tripkovic, D.; Strmenik, D.; Chang, K. C.; Uchimura, M.; Paulikas, A. P.; Stamenkovic, V.; Markovic, N. M. Science 2011, 334, 1256-1260.

(18) Yin, H.; Zhao, S.; Zhao, K.; Muqsit, A.; Tang, H.; Chang, L.; Zhao, H.; Gao, Y.; Tang, Z. Nat. Commun. 2015, 6, 6430.

(19) Gong, M.; Zhou, W.; Tsai, M. C.; Zhou, J.; Guan, M.; Lin, M. C.; Zhang, B.; Hu, Y.; Wang, D. Y.; Yang, J.; Pennycook, S. J.; Hwang, B. J.; Dai, H. Nat. Commun. 2014, 5, 4695.

(20) Wang, L.; Lin, C.; Huang, D.; Chen, J.; Jiang, L.; Wang, M.; Chi, L.; Shi, L.; Jin, J. ACS Catal. 2015, 5, 3801-3806.

(21) Zhuang, Z.; Giles, S. A.; Zheng, J.; Jenness, G. R.; Caratzoulas, S.; Vlachos, D. G.; Yan, Y. Nat. Commun. 2016, 7, 10141.

(22) Imbihl, R.; Behm, R. J.; Ertl, G.; Moritz, W. Surf. Sci. 1982, 123, 129-140.

(23) Tran, P. D.; Tran, T. V.; Orio, M.; Torelli, S.; Truong, Q. D.; Nayuki, K.; Sasaki, Y.; Chiam, S. Y.; Yi, R.; Honma, I.; Barber, J.; Artero, V. Nat. Mater. 2016, 15, 640-646.

(24) Torella, J. P.; Gagliardi, C. J.; Chen, J. S.; Bediako, D. K.; Colón, B.; Way, J. C.; Silver, P. A.; Nocera, D. G. Proc. Natl. Acad. Sci. U. S. A. 2015, 112, 2337-2342.

(25) Cobo, S.; Heidkamp, J.; Jacques, P. A.; Fize, J.; Fourmond, V.; Guetaz, L.; Jousselme, B.; Ivanova, V.; Dau, H.; Palacin, S.; Fontecave, M.; Artero, V. Nat. Mater. 2012, 11, 802-807.
(26) Miao, J.; Xiao, F. X.; Yang, H. B.; Khoo, S. Y.; Chen, J.; Fan, Z.; Hsu, Y. Y.; Chen, H. M.; Zhang, H.; Liu, B. Sci. Adv. 2015, $1, \mathrm{e} 1500259$

(27) You, B.; Sun, Y. Adv. Energy Mater. 2016, 6, 1502333.

(28) Conway, B. E.; Tilak, B. V. Electrochim. Acta 2002, 47, 35713594.

(29) Lu, Q.; Hutchings, G. S.; Yu, W.; Zhou, Y.; Forest, R. V.; Tao, R.; Rosen, J.; Yonemoto, B. T.; Cao, Z.; Zheng, H.; Xiao, J. Q.; Jiao, F.; Chen, J. G. Nat. Commun. 2015, 6, 6567.

(30) Greeley, J.; Jaramillo, T. F.; Bonde, J.; Chorkendorff, I.; Nørskov, J. K. Nat. Mater. 2006, 5, 909-913.

(31) Subbaraman, R.; Tripkovic, D.; Chang, K. C.; Strmenik, D.; Paulikas, A. P.; Hirunsit, P.; Chan, M.; Greeley, J.; Stamenkovic, V.; Markovic, N. M. Nat. Mater. 2012, 11, 550-557.

(32) You, B.; Sun, Y. ChemPlusChem 2016, 81, 1045-1055.

(33) Gong, Q.; Wang, Y.; Hu, Q.; Zhou, J.; Feng, R.; Duchesne, P. N.; Zhang, P.; Chen, F.; Han, N.; Li, Y.; Jin, C.; Li, Y.; Lee, S. T. Nat. Commun. 2016, 7, 13216.

(34) Ravel, B.; Newville, M. J. Synchrotron Rad. 2005, 12, 537-541.

(35) Kresse, G.; Furthmüller, J. Phy. Rev. B 1996, 54, 11169-11186.

(36) Blöchl, P. E. Phy. Rev. B 1996, 50 17953-17979.

(37) Perdew, J. P.; Burke, K.; Ernzerhof, M. Phys. Rev. Lett. 1996, 77, 3865-3868.

(38) Henkelman, G.; Uberuaga, B. P; Jónsson, H. J. Chem. Phys. 2000, 113, 9901-9904. 
Insert Table of Contents artwork here

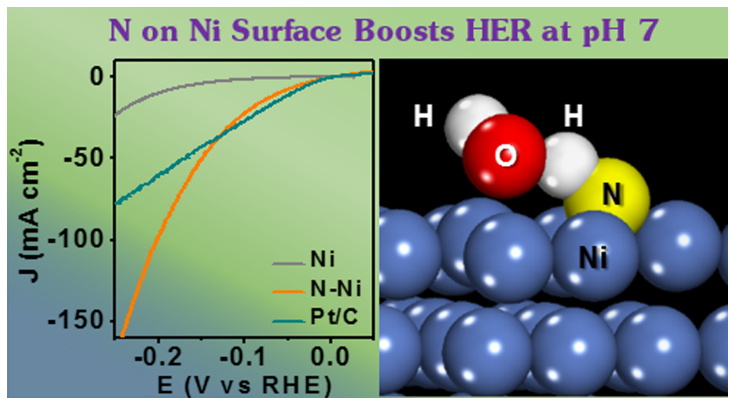

